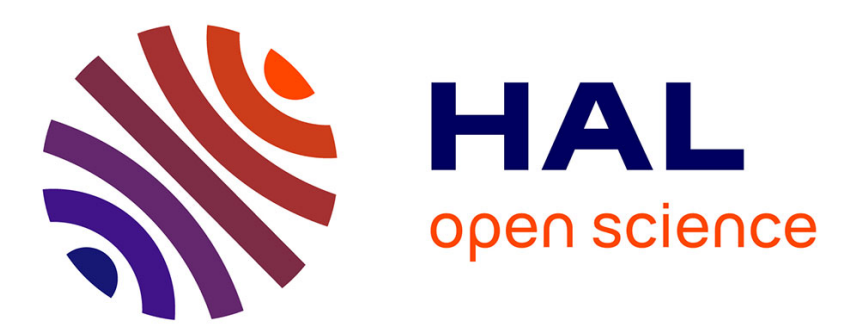

\title{
Multipoint phase calibration for improved compensation of inherent wavefront distortion in parallel aligned liquid crystal on silicon displays
}

\author{
Joaquín Otón, Pierre Ambs, María S. Millán, Elisabet Pérez-Cabré
}

\section{- To cite this version:}

Joaquín Otón, Pierre Ambs, María S. Millán, Elisabet Pérez-Cabré. Multipoint phase calibration for improved compensation of inherent wavefront distortion in parallel aligned liquid crystal on silicon displays. Applied optics, 2007, 46 (23), pp.5667-5679. 10.1364/AO.46.005667 . hal-00878836

\author{
HAL Id: hal-00878836 \\ https://hal.science/hal-00878836
}

Submitted on 31 Oct 2013

HAL is a multi-disciplinary open access archive for the deposit and dissemination of scientific research documents, whether they are published or not. The documents may come from teaching and research institutions in France or abroad, or from public or private research centers.
L'archive ouverte pluridisciplinaire HAL, est destinée au dépôt et à la diffusion de documents scientifiques de niveau recherche, publiés ou non, émanant des établissements d'enseignement et de recherche français ou étrangers, des laboratoires publics ou privés. 


\title{
Multipoint phase calibration for improved compensation of inherent wavefront distortion in parallel aligned liquid crystal on silicon displays
}

\author{
Joaquín Otón, ${ }^{1}$ Pierre Ambs, ${ }^{2}$ María S. Millán, ${ }^{1, \star}$ and Elisabet Pérez-Cabré ${ }^{1}$ \\ ${ }^{1}$ Department of Optics and Optometry, Technical University of Catalonia, Violinista Vellsolà 37, \\ Terrassa (Barcelona) 08222, Spain \\ 2Laboratoire Modélisation Intelligence Processus Systèmes, École Nationale Supérieure d'Ingénieurs Sud \\ Alsace-Mulhouse, Université de Haute Alsace, 12 rue des Frères Lumière, Mulhouse Cedex 68093, France \\ ${ }^{\star}$ Corresponding author: millan@oo.upc.es \\ Received 8 March 2007; accepted 10 April 2007; \\ posted 23 April 2007 (Doc. ID 80779); published 8 August 2007
}

\begin{abstract}
The inherent distortion of a reflective parallel aligned spatial light modulator (SLM) may need compensation not only for the backplane curvature but also for other possible nonuniformities caused by thickness variations of the liquid crystal layer across the aperture. First, we build a global look-up table (LUT) of phase modulation versus the addressed gray level for the whole device aperture. Second, when a lack of spatial uniformity is observed, we define a grid of cells onto the SLM aperture and develop a multipoint calibration. The relative phase variations between neighboring cells for a uniform gray level lead us to build a multi-LUT for improved compensation. Multipoint calibration can be done using either phase-shift interferometry or Fourier diffraction pattern analysis of binary phase gratings. Experimental results show the compensation progress in diffractive optical elements displayed on two SLMs. (C) 2007 Optical Society of America

OCIS codes: $\quad 220.1000,050.1970,230.6120,230.3720$.
\end{abstract}

\section{Introduction}

Flexibility and programmability of spatial light modulators (SLMs) add numerous advantages in optical systems. Their technical improvement permits widespread use in applications such as reconfigurable optical interconnects [1,2], programmable lenses and filters [3-7], optical tweezers [8], particle trapping, [9], optical metrology [10,11], holographic 3D displays [12], dynamic displays [13], adaptive optics [14-16], and aberration compensation [17-19].

A good and complete calibration is an essential step in the electrically addressed SLM initialization, particularly if the SLM is to be used in applications that require high wavefront control. Every single SLM unit may show significant differences in its optical behavior from other units of even the same trademark and model [20]. It is clear that the quality and

0003-6935/07/235667-13\$15.00/0

(C) 2007 Optical Society of America effectiveness of the optical component addressed to the SLM strongly depend on the knowledge of the device response. In fact, the signal must be modified before addressing it to the SLM to compensate for the distortions internally introduced by the device and, hence, to eventually reproduce the optical component with the desired performance.

Many calibration methods of transmissive and reflective SLM have been reported. Osten and coworkers reviewed several types of modern SLM, methods for their characterization, as well as some recent applications [11].

A group of calibration methods [21-27] considers a general parameterization of the Jones matrix of a twisted-nematic liquid crystal display (LCD), with the director axis of the liquid crystal oriented in a given direction at the input surface of the display. In the calibration setup, the LCD is placed between two linear polarizers working as polarizer and analyzer. The values of the intensity transmitted by the system for different configurations of the polarizer and the 
LCD are used to calculate the parameters that characterize the LCD performance in the Jones matrix formalism. Since the amplitude and phase modulations are usually coupled in a transmissive twistednematic LCD, the calibration of the display allows one to select the polarization configuration, the polarization state of impinging light, and the wavelength to obtain either amplitude-mostly, or phasemostly, or amplitude-phase coupled modulation with an optimized dynamic range. Particularly for those LCDs that do not reach $2 \pi$ phase modulation it is important to obtain adequate dynamic ranges and try to correct for the nonlinear mapping of digital graylevel (voltage) to phase modulation [20,28-31].

Another group of calibration methods focuses on the spatial uniformity of the LCD response. Transmissive LCDs have commonly been assumed to have a spatially uniform response except for the work described in Ref. [32]. However, in the case of a reflective liquid crystal on silicon (LCoS) SLM, inherent curvatures of the device surface, mainly because of limitations in the polishing process of the silicon backplane, cause reflective wavefront distortion in the optical system $[20,28,30]$. Fringe analysis using an interferometer is a common procedure to measure this wavefront distortion [20,30,33-35]. The nonuniformity may also appear as an additional effect because of the curvature of the cover glass, causing differences in thickness of the liquid crystal layer $[16,30]$. New testing methods are being designed for a more complete calibration adapted to each SLM and, along with them, new algorithms are being developed to self-compensate for the phase distortions introduced by the device. New control software is already provided by manufacturers to allow users to correct the distortion introduced by the backplane curvature without significantly affecting the dynamic range $[20,36]$.

However, the compensation of the aberration introduced by the backplane curvature can sometimes be insufficient. As it has been pointed out with the cover glass, the lack of spatial uniformity of the SLM surface can have other reasons such as thickness variations of the liquid crystal because of bending of the supporting sheets in the fabrication procedure [16].

In this paper we deal with the spatial calibration of reflective LCoS SLM by testing the aperture of the device to characterize the inherent phase distortion. We propose a compensation of this inherent distortion that involves not only the compensation for the backplane curvature but also, when it is necessary, an additional compensation for other possible sorts of nonuniformities caused by thickness variations of the liquid crystal layer across the aperture of the SLM. We assume that the extraordinary index depends on the addressed gray level (voltage) and, for a given value of the gray level, the extraordinary index is constant in all points of the display aperture. We will define a grid of elemental square cells onto the SLM aperture and develop a multipoint calibration by means of which the relative phase variations between neighboring elemental cells for a uniform gray level addressed to them (same voltage) are detected. From the measurement of these variations, an updated map of the inherent phase error can be built and a multi look-up table (LUT) can be defined for more complete compensation. Our method will generate a specific LUT for every element of the SLM aperture in accordance with the technique reported by Xun and Cohn [30]. Following a four-phase phase-shift interferometry algorithm [37], Xun and Cohn captured four interferograms for each gray level value and computed the phase at any location of the device aperture. Their procedure had to meet strict experimental requirements such as the control of four successive small $(\lambda / 4)$ displacements of the reference mirror for each gray level value and the reduction of the elapsed time between recordings to minimize vibration errors in interferometric measurements by gathering the phase data as quickly as possible. Our method, however, can be applied in more relaxed experimental conditions, as we will show in Subsection 3.C. The multipoint calibration we propose can be applied on the basis of either phase-shift interferometry or Fourier diffraction pattern analysis of binary phase gratings. In the latter case, we would apply the method proposed by Zhang et al. [38] to each cell defined on the SLM aperture.

We deal with two reflective parallel aligned nematic SLMs whose phase modulation capability exceeds $2 \pi$ in the visible range and no coupled amplitude modulation has been detected (Tables 1 and 2 ). In the first stage, we proceed with an approximated calibration of the LCoS SLM. This calibration allows us to build a global LUT for the whole aperture. The global LUT maps the 8-bit range of gray scale values over a full wave, with phase values from 0 to $2 \pi$, in such a way that a linear phase response of the SLM is obtained, in first approach, by using mapped gray values that linearly increase from 0 to 255. Two methods are used to obtain this global LUT: one is based on converting gray scale differences to phase shifts and measuring them in a Michelson interferometer [20], and the other is based on the afore-

Table 1. Technical Specifications of the HEO-1080 P SLM from Holoeye

\begin{tabular}{ll}
\hline \multicolumn{1}{c}{ Parameters } & \multicolumn{1}{c}{ Specifications } \\
\hline Brand and model & $\begin{array}{l}\text { Holoeye HEO 1080 P (2006) } \\
\text { Device configuration }\end{array}$ \\
& $\begin{array}{c}\text { Reflective parallel aligned } \\
\text { nematic liquid crystal }\end{array}$ \\
Array size & $15.36 \times 8.64(\mathrm{~mm} \times \mathrm{mm})$ \\
Resolution & $1920(\mathrm{H}) \times 1080(\mathrm{~V})$ \\
Pixel pitch & $8 \mu \mathrm{m}$ \\
Fill factor & $>87 \%$ \\
Max. phase modulation & $2.8 \pi$ \\
$\quad(\lambda=633 \mathrm{~nm})$ & \\
Max. coupled amplitude & $<5 \%$ \\
$\quad$ mod. $(\lambda=633 \mathrm{~nm})$ & \\
Zero-order diffraction & $60 \%$ \\
$\quad$ efficiency & \\
Maximum usable frame rate & $60 \mathrm{~Hz}$ \\
Electrical addressing & $8-\mathrm{bit}$ via DVI interface \\
\hline
\end{tabular}


Table 2. Technical Specifications of the P256 ZTN-LC SLM from BNS

\begin{tabular}{|c|c|}
\hline Parameters & Specifications \\
\hline Brand and model & BNS P256 (2003) \\
\hline Device configuration & $\begin{array}{l}\text { Reflective parallel aligned } \\
\text { nematic liquid crystal }\end{array}$ \\
\hline Array size & $4.608 \times 4.608(\mathrm{~mm} \times \mathrm{mm})$ \\
\hline Resolution & $256 \times 256$ \\
\hline Pixel pitch & $18 \mu \mathrm{m}$ \\
\hline Fill factor & $85 \%$ \\
\hline $\begin{array}{l}\text { Max. phase modulation } \\
\quad(\lambda=633 \mathrm{~nm})\end{array}$ & $2 \pi$ \\
\hline $\begin{array}{l}\text { Max. coupled amplitude } \\
\text { mod. }(\lambda=633 \mathrm{~nm})\end{array}$ & $<3 \%$ \\
\hline $\begin{array}{l}\text { Zero-order diffraction } \\
\text { efficiency }\end{array}$ & $65 \%$ \\
\hline Optical flatness & $\lambda / 3$ \\
\hline Maximum usable frame rate & $25-150 \mathrm{~Hz}^{a}$ \\
\hline Electrical addressing & 8-bit \\
\hline Driver memory & 4096 frames of SDRAM \\
\hline
\end{tabular}

${ }^{a}$ Depends on phase stroke, wavelength, and temperature.

mentioned display of binary phase gratings [38]. When a lack of spatial uniformity is observed, the wavefront distortion on the LCoS SLM is measured and interpreted by analyzing the interference fringes. We use a wavefront decomposition in Zernike polynomials that has been demonstrated to be useful in evaluating and compensating for the backplane curvature [20]. Because of the circular definition of the Zernike polynomials, the compensation will not reach the corners of a SLM with square or rectangular aperture. The multipoint calibration can be particularly useful when the wavefront distortion has no circular symmetry. From the multipoint calibration, a multi-LUT can be built and conveniently smoothed by interpolation. In our experiments, we will find that one of the analyzed LCoS SLMs requires the multipoint calibration and the multi-LUT to improve the mapping of gray levels over a full wave. On the other hand, both modulators require compensation of inherent wavefront distortion. In the case of a modulator with multipoint calibration, the compensation as well as other programmed phase distributions will be more effectively reproduced by using the multi-LUT. Experimental results that give evidence of the compensation progress are provided and discussed. We discuss the results obtained when the SLM is used to display diffractive optical elements (DOEs), with and without compensation of inherent wavefront distortion, and either with global LUT or with multi-LUT.

\section{Technical Characteristics of the Spatial Light Modulators}

The study of the calibration is focused on two SLMs working as reflective displays that are based on the parallel aligned LCoS technology. One of them is the very recent HEO $1080 \mathrm{P}$ model manufactured by Holoeye in 2006 [36]. The other is the P256 Zero-Twist Nematic Liquid Crystal (ZTN-LC) model manufac- tured by Boulder Nonlinear Systems (BNS) in 2003 [39]. Both modulators are used in a pure phase modulation regime. Apparently, the main differences between the technical features of both displays are their pixel resolution and pixel pitch. The Holoeye panel has $1920 \times 1080$ pixels in a $15.36 \mathrm{~mm} \times 8.64 \mathrm{~mm}$ array, with $8.0 \mu \mathrm{m}$ pixel pitch, $87 \%$ fill factor, $60 \%$ zero order diffraction efficiency, and 8-bit electrically addressable levels. The BNS device is a $256 \times 256$ pixel array of $4.608 \mathrm{~mm} \times 4.608 \mathrm{~mm}$ size, with $18 \mu \mathrm{m}$ pixel pitch, $85 \%$ fill factor, $65 \%$ zero order diffraction efficiency, and 8-bit electrically addressable levels. Other features of these modulators are specified in Tables 1 and 2.

If dynamic characteristics of the displays are to be considered, these modulators have other relevant differences, mainly because of the way images are addressed to the displays. On the one hand, the Holoeye panel has a fixed frame rate of $60 \mathrm{~Hz}$. On the other hand, the BNS model has a frame rate frequency up to $150 \mathrm{~Hz}$, according to technical specifications. This range permits us to consider the BNS model as a device with one of the highest frequency frame rates.

\section{Phase Calibration}

\section{A. Phase Calibration Based on Phase Shift Interferometry}

Since the parallel aligned LCoS SLMs are to be used as phase-only modulators of linearly polarized light beams, with the direction of polarization coincident with the extraordinary axis, there is no essential need to measure the parameters of the Jones matrix of the devices, and we focus on the characterization of their phase shifting capability. To experimentally measure the phase modulation of the SLMs, we initially considered the conceptually simple method based on analysis of the interference fringes obtained in a Michelson interferometer (Fig. 1). We used a reference mirror flatter than $\lambda / 20$ and, in the initialization stage of the setup, we verified that the beam splitter (cube) did not significantly modify the polarization state of the light. The image addressed to the device consists of two sectors, whose respective gray

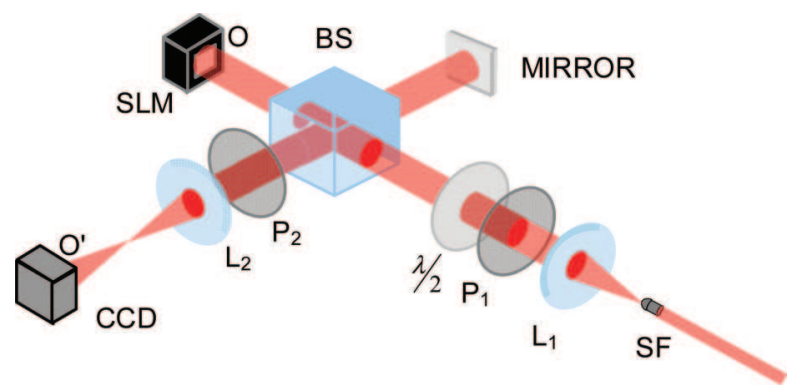

Fig. 1. (Color online) Michelson interferometer to measure the spatial uniformity and the phase modulation capability of the SLM: $\mathrm{P}_{1}$ and $\mathrm{P}_{2}$, linear polarizers, $\mathrm{L}_{1}, \mathrm{~L}_{2}$, lenses. $\mathrm{SF}$ and $\mathrm{BS}$ stand for spatial filter and beam splitter, respectively. A halfwave plate (denoted $\lambda / 2$ ) is used to adjust the polarization of the incident beam on the SLM. The SLM plane $(\mathrm{O})$ is imaged onto the CCD sensor $\left(\mathrm{O}^{\prime}\right)$. 


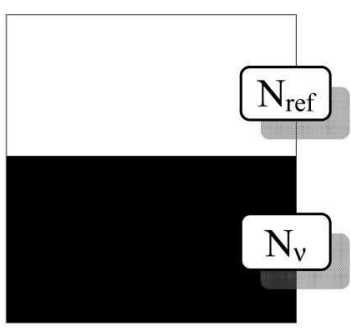

(a)

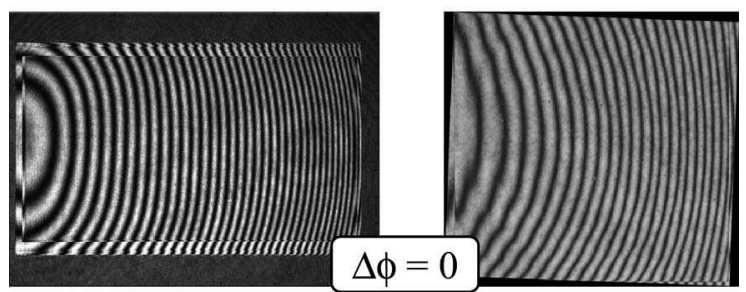

(b)

(e)

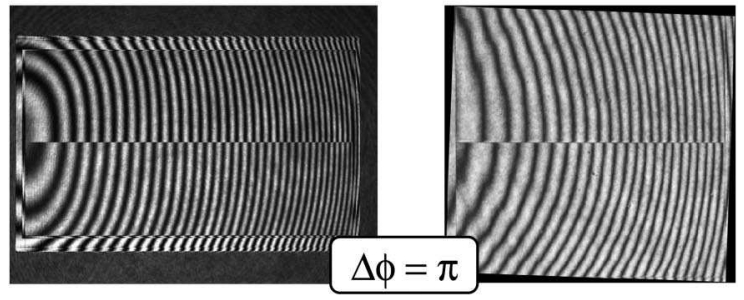

(c)

(f)

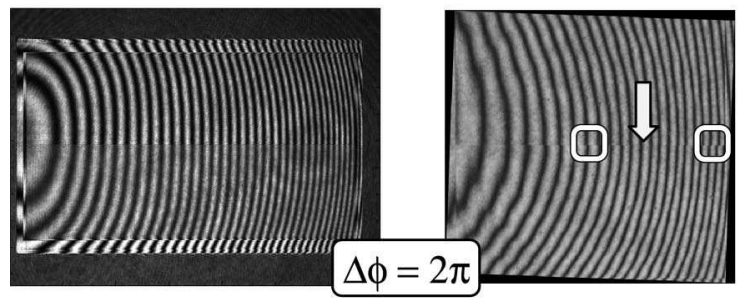

(d)

(g)

Fig. 2. (a) Image consisting of two sectors with the reference gray level $N_{\text {ref }}$ and the varying gray level $N_{v}$; (b)-(d) interferograms of the Holoeye modulator obtained with $\Delta \phi(N) \approx 0, \pi, 2 \pi$, respectively; (e)-(g) interferograms of the BNS modulator obtained with $\Delta \phi(N) \approx 0, \pi, 2 \pi$, respectively.

levels are the reference gray level $N_{\text {ref }}$ and the varying gray level $N_{v}$ [Fig. 2(a)]. The LCoS SLM converts the gray level difference into a measurable phase shift $\Delta \phi(N)=\phi\left(N_{v}\right)-\phi\left(N_{\text {ref }}\right)$. If the reference mirror of the interferometer is gently tilted, the interferogram intensity pattern shows two shifted series of fringes corresponding to the two sectors of the SLM aperture. The measurement of the fringe shift is proportional to the phase shift on modulo $2 \pi$. This is true provided the SLM has a spatially uniform response and, therefore, for any pair of sectors with addressed gray level values $\left(N_{v}, N_{\text {ref }}\right)$, the device displays a single value of phase shift along the border. For example, Figs. 2(b)-2(d) show the experimental results corresponding to the Holoeye modulator for $\Delta \phi(N)$ $\approx 0, \pi, 2 \pi$, respectively. It can be seen that the fringe

shift stays approximately constant along the horizontal axis. This result gives evidence of a uniform response along the axis. The Holoeye modulator showed a spatially uniform response in the first approach. The method fails when the SLM response is not spatially uniform, i.e., when the device displays different phase shifts for a given pair of addressed gray level values $\left(N_{v}, N_{\text {ref }}\right)$. For example, Figs. 2(e)2 (g) show the experimental results corresponding to the BNS modulator for $\Delta \phi(N) \approx 0, \pi, 2 \pi$, respectively. It can be seen that the fringe shift does not keep constant along the horizontal axis. For instance, for $\Delta \phi(N) \approx 2 \pi$ in Fig. 2(g), the arrow points to the region where there is a real $2 \pi$ modulation. Meanwhile, the fringes in the center and the border are not aligned, because of a different phase modulation. This result gives evidence of the lack of uniformity in the device response along the axis. This irregular response was already observed by Xun and Cohn [30] for a $512 \times 512$ BNS modulator.

This method can be used to calibrate the Holoeye modulator, but not the BNS modulator, as we will see in the following.

\section{Characterization of Holoeye HEO 1080P Phase Modulation}

This modulator is electrically addressed by means of a standard digital video interface (DVI) connector and works with a specific 22-6 bit-plane configuration, which allows mapping the 256 video gray levels to 1472 index levels of the LUT loaded in the hardware of the device. Using phase-shifting interferometry, we measured the phase modulation capability of the Holoeye modulator. Figure 3(a) shows the phase shift modulation for the 1472 index levels using a $\mathrm{He}-\mathrm{Ne}$ laser with $\lambda=633 \mathrm{~nm}$. It can be seen that the modulation range is more than $3 \pi$ (slighty more than what it corresponds to in the technical specifications in Table 1). Since in most of the applications, such as dynamic DOEs or adaptive optics, there is a need for a phase modulation range of $[0,2 \pi]$, it is important to build an LUT that maps the 8-bit video gray level range $[0,255]$ into a phase modulation range $[0,2 \pi]$ and linearizes the response. We have selected the range limited by the index levels 200 and 680 for which there is a phase shift of $2 \pi$ in Fig. 3(a). We depict the obtained LUT in Fig. 3(b) and the phase modulation in Fig. 3(c). From the index level range selected it can be said that there are 256 gray levels that can be effectively addressed by the modulator hardware with no further restrictions on the number of addressable gray levels.

\section{B. Phase Calibration Based on Binary Phase Gratings}

We tried an alternative method to calibrate the spatially nonuniform BNS SLM. The method was proposed by Zhang et al. [38] and is based on displaying binary phase (Ronchi) gratings and measuring the intensities in the diffraction pattern at the Fourier plane (Fig. 4). A diffraction binary grating of square fringes with gray level values given by the reference gray level $N_{\text {ref }}$ and the varying gray level $N_{v}$ is ad- 


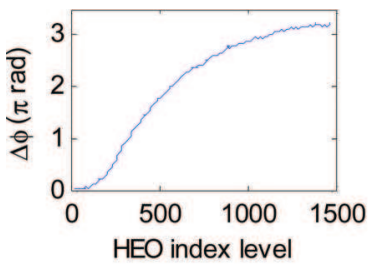

(a)

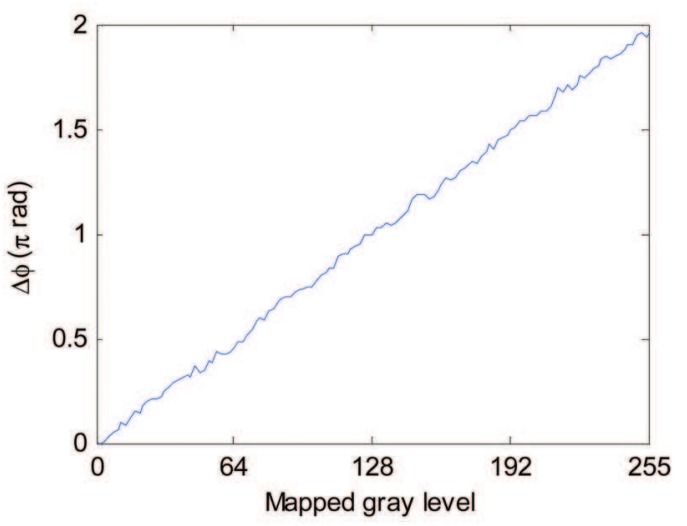

(c)

Fig. 3. (Color online) (a) Phase modulation for the Holoeye HEO $1080 \mathrm{P}$ SLM as a function of the index level range $[0,1471]$ for the illuminating wavelength $633 \mathrm{~nm}$. (b) LUT to map the 8-bit addressed gray levels on the index level range of $[200,680]$ that corresponds to a phase modulation of $2 \pi$ measured in (a). (c) Linear phase modulation obtained using the LUT of (b).

dressed to be displayed on the SLM. Let us denote $\Delta \phi(N)=\phi\left(N_{v}\right)-\phi\left(N_{\text {ref }}\right)$ as the phase shift corresponding to the gray levels $N_{v}$ and $N_{\text {ref. }}$. It is assumed that the coupled amplitude modulation is negligible as it corresponds to a pure phase grating. The intensity at the first diffraction order is proportional to

$$
I_{1}(\Delta \phi) \propto[1-\cos \Delta \phi(N)]
$$

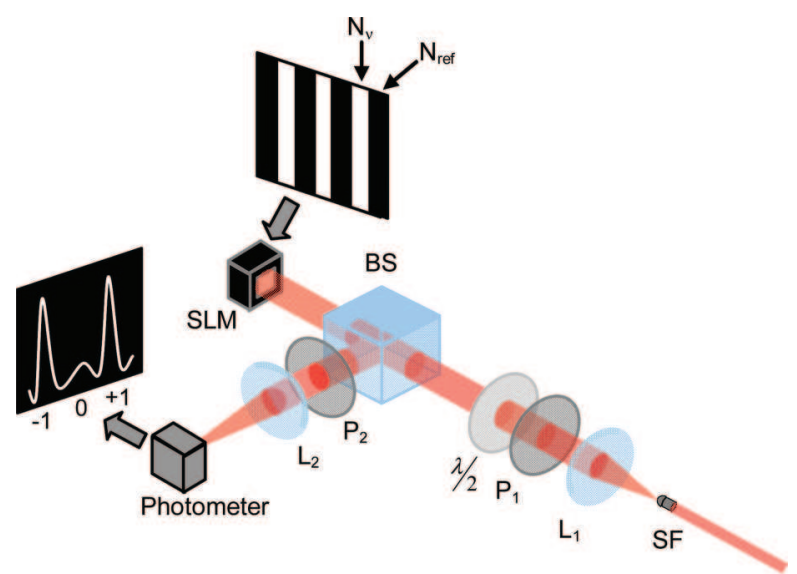

Fig. 4. (Color online) Setup for the calibration of the BNS SLM, based on the display of binary phase Ronchi gratings on the SLM and the analysis of first order peak intensity of the Fourier diffraction pattern. The gray level values of the grid are $N_{\text {ref }}$ and $N_{v}$.
The intensity function of Eq. (1) shows a maximum value for phase shifts that are odd multiples of $\pi$ and a minimum value for those phase shifts multiples of $2 \pi$. The wavelength used in the calibration of the BNS modulator was $633 \mathrm{~nm}$ from a He-Ne laser. The gratings displayed had a spatial frequency of two pixels/period in the horizontal direction of the SLM (according to the orientation of the BNS SLM shown in the figures). The reference gray level was set to $N_{\text {ref }}=128$ because this value was equivalent to the off state. The experimental results obtained for the intensity of the first diffraction order $I_{1}(\Delta \phi)$ are plotted in Fig. 5(a). It can be seen that the liquid crystal device performs symmetrically around the gray level $N_{v}=128$. The response of the liquid crystal does not depend on the polarity of the applied voltage and therefore the phase corresponding to gray level $N_{v}$ is equal to the phase corresponding to gray level 255 $-N_{v}$. Although the shape of the function is not a perfect sinusoid, the maximum peak value can be associated with a phase shift of $\Delta \phi(N)=\pi$. On both sides of this maximum, we take the two minimum values to fix the range limits $\Delta \phi(N)=0$ and $\Delta \phi(N)$ $=2 \pi$ [Fig. 5(a)]. From Fig. 5(a) we plot the phase shift versus the gray level in Fig. 5(b), where it can be seen that the phase modulation range achieved by the BNS SLM reaches $2.3 \pi$. Initially, we limit the phase modulation range to $[0,2 \pi]$ by tentatively limiting the index level range to $[0,90]$. To linearize the phase response we built an LUT. For practical reasons, this LUT was also built to extend the index level range $[0,90]$ into a more common range of 8-bit (256) mapped gray levels [Fig. 5(c)]. Using this new LUT, the intensity experimentally measured for the first diffraction order $I_{1}(\Delta \phi)$ [Fig. $5(\mathrm{~d})$ ] recovers the sinusoidal appearance.

The method used here has allowed us to calibrate the phase response of the BNS SLM on average on the device aperture. The gray level that reaches a maximum value of $I_{1}(\Delta \phi)$ in Fig. 5(a) does not necessarily imply a phase shift of $\pi$ in all the points of the Ronchi grating displayed on the SLM aperture. Although the gray level image addressed to the modulator is binary with $N_{\text {ref }}, N_{v}$ values, the displayed phase grating on the spatially nonuniform BNS SLM has different phase shifts depending on the zone of the aperture. For this reason, it is convenient to obtain a more precise result with the multipoint calibration we show in the next section.

\section{Multipoint Calibration}

Taking into consideration a previous result of Xun and Cohn [30], in which the phase modulation of their BNS ZT-LC $512 \times 512$ modulator was larger near the border than in the center of the aperture, we tried to check this characteristic in our BNS modulator. Again using phase-shift interferometry and fringe analysis, we measured the phase modulation of two small regions, one in the center and another in the border of the aperture. The experimental results are plotted in Fig. 6(a) versus the gray level addressed in the BNS index level range [0,100]. For the sake of 


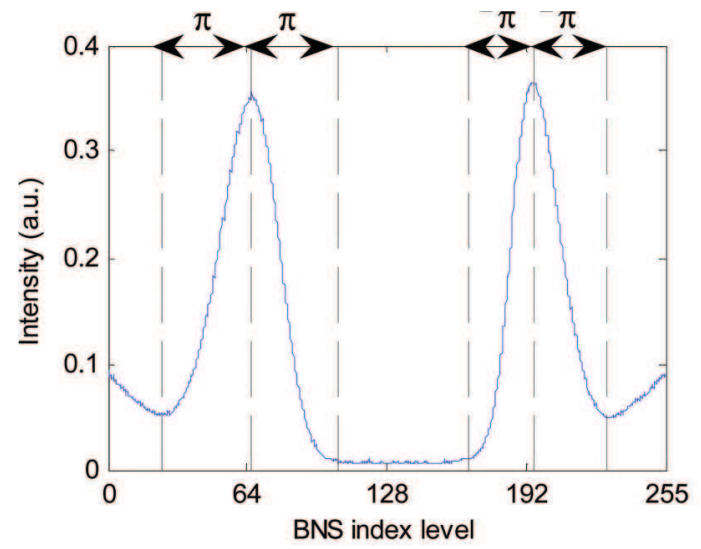

(a)

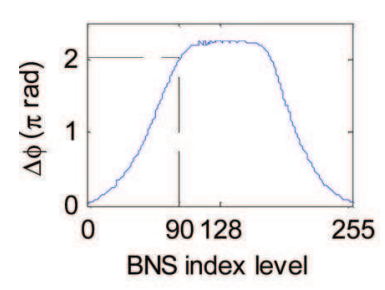

(b)

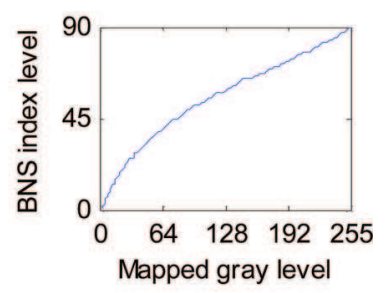

(c)

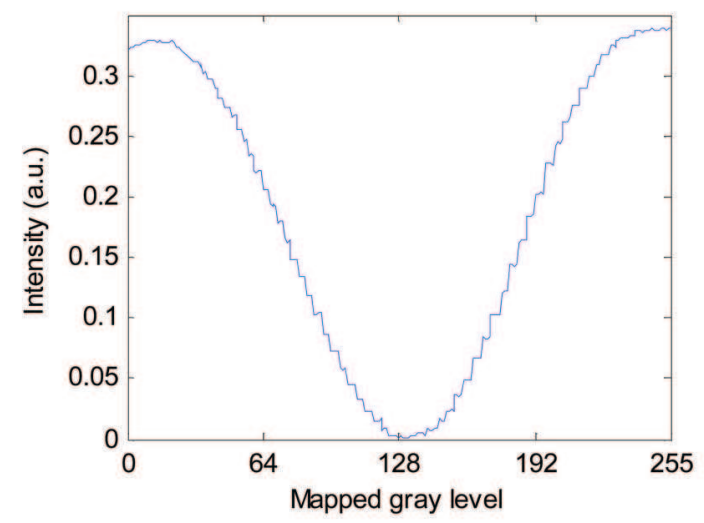

(d)

Fig. 5. (Color online) Calibration of the BNS SLM based on displaying binary phase Ronchi gratings. (a) Intensity for the first diffraction order $I_{1}(\Delta \phi)$ as function of the index level $N_{v}$ varying from 0 to 255. (b) Phase shift computed from the data plotted in (a). (c) LUT used to extend the index level range $[0,90]$ to a mapped 8-bit gray level range $[0,255]$ and equivalently map a lineal phase shift range modulation $[0,2 \pi]$. (d) Intensity of the first diffraction order $I_{1}(\Delta \phi)$ of (a) as a function of the mapped 8-bit gray level range $[0,255]$.

comparison, the curves of the phase modulation in the center and in the border are plotted along with the curve obtained by the Ronchi phase grating method. We see in Fig. 6(a) that for an index level of 90 the phase modulation in the border is around $2.5 \pi$ rads, while in the center of the aperture the phase modulation is close to but does not reach $2 \pi$. For this reason, to reach a phase modulation of $2 \pi$ in the center we finally extended the range of index level to $[0,100]$. The curve obtained by displaying a Ronchi phase grating shows that the average phase modula-

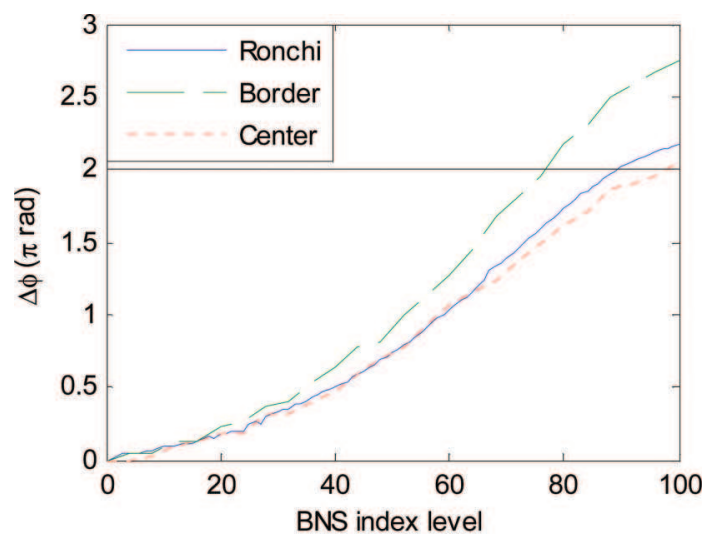

(a)

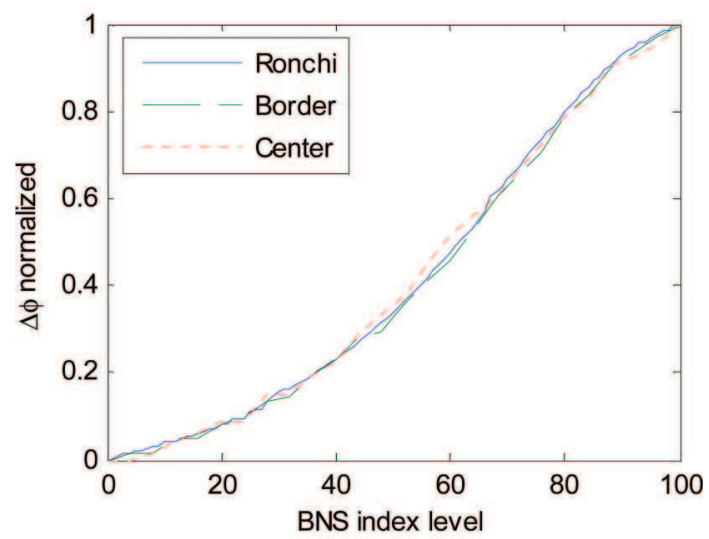

(b)

Fig. 6. (Color online) (a) Plots of the phase shift vs. the BNS index level in the central area of SLM aperture (dotted), at a border region (dashed), and that obtained by the phase Ronchi grating method (solid). (b) Phase shift curves plotted in (a) normalized to their maximum values in the index level range $[0,100]$.

tion of the device is closer to the response in the center than in the border.

Because of the birefringence exhibited by the liquid crystal, the relative phase retardation introduced in the wave propagation can be written as

$$
\Delta \phi(x, y, N)=\frac{2 \pi}{\lambda}\left(n_{e}(x, y, N)-n_{0}\right) d(x, y),
$$

where $d(x, y)$ is the path of light within the liquid crystal, that is, twice the thickness of the liquid crystal layer for a given point $(x, y)$ of the device aperture, $n_{0}$ is the ordinary index, and $n_{e}$ is the extraordinary index, which depends on the addressed gray level $N$. Assuming that the extraordinary index is spatially constant, that is,

$$
n_{e}(x, y, N)=n_{e}(N) \forall(x, y),
$$

Eq. (2) can be rewritten as

$$
\Delta \phi(x, y, N)=\frac{2 \pi}{\lambda}\left[n_{e}(N)-n_{0}\right] d(x, y),
$$


which implies that for a given gray level value of $N$ the phase difference between any two points of the aperture is because of the thickness variation between them. Let us associate the phase modulation curves of Fig. 6(a) with the retardation and let us normalize them by dividing each curve by its maximum value in the gray level range:

$$
\begin{aligned}
\Delta \phi_{\text {Norm }}(x, y, N)=\frac{\Delta \phi(x, y, N)}{\Delta \phi_{\text {Max }}\left(x, y, N^{\prime}\right)} & =\frac{n_{e}(N)-n_{0}}{n_{e}\left(N^{\prime}\right)-n_{0}} \\
& =\Delta \phi_{\text {Norm }}(N) .
\end{aligned}
$$

The constant of normalization has been arbitrarily taken at $\Delta \phi_{\mathrm{Max}}\left(x, y, N^{\prime}\right)$, where $N^{\prime}$ is the gray level for which $\Delta \phi$ is maximum. For a given point $(x, y)$ of the aperture, the $\Delta \phi_{\text {Norm }}(N)$ function depends on the variation of the extraordinary index with the gray level $N$. The curves of Fig. 6(a) appear normalized in Fig. 6(b). If we compare the three curves, we realize that the corresponding normalized retardations are coincident. Thus, this confirms the assumption that the lack of spatial uniformity in the phase retardation is basically because of the thickness variations across the SLM aperture. This result is consistent with that previously reported by Xun and Cohn [30]. We will take advantage of this result to calibrate the phase modulation in any point of the SLM aperture from the already measured normalized retardation $\Delta \phi_{\text {Norm }}(N)$. Thus, if we denote $N_{2 \pi}(x, y)$ the gray level corresponding to a $2 \pi$ phase modulation in the position $(x, y)$ and take into account Eq. (5), we can write

$$
\Delta \phi_{\mathrm{Max}}(x, y)=\frac{2 \pi}{\Delta \phi_{\mathrm{Norm}}\left[N_{2 \pi}(x, y)\right]},
$$

and hence, the phase retardation can be rewritten as

$$
\Delta \phi(x, y, N)=\Delta \phi_{\text {Norm }}(N) \frac{2 \pi}{\Delta \phi_{\text {Norm }}\left[N_{2 \pi}(x, y)\right]} .
$$

From Eq. (7) and the already determined $\Delta \phi_{\text {Norm }}(N)$ [Fig. 6(b)], we only need to find the gray level $N_{2 \pi}(x, y)$ in a given point $(x, y)$ to estimate $\Delta \phi(x, y, N)$. In this way, the calibration of the phase modulation and the corresponding LUT can be built for any point of the device aperture. In practice, however, we did not calibrate the phase modulation capability of every pixel but, instead, we sampled the SLM aperture taking $8 \times 8$ square cells [Fig. 7(a)]. For each cell, we determined the value $N_{2 \pi}$ by introducing the proper phase shift in the interference fringes of the Michelson interferometer. In the experimental procedure, we displayed a sort of phase chessboard setting alternate cells to $N_{\text {ref }}=0$ [Fig. 7(b)]. Looking preferably at the surroundings of a vertex of a given sector, we visually searched the gray level $N_{v}=N_{2 \pi}$ that made the fringe series move just one fringe to obtain a perfect match

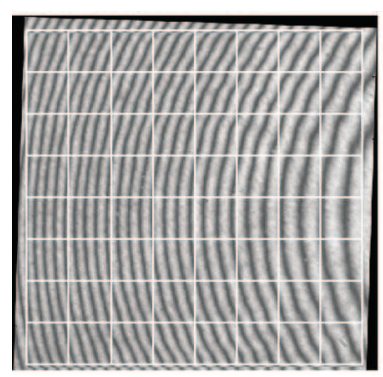

(a)

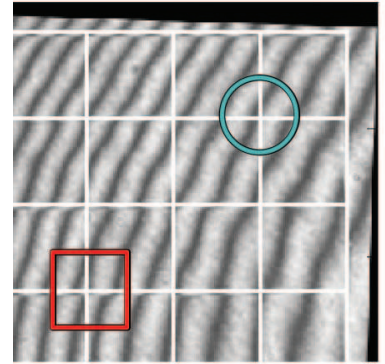

(c)

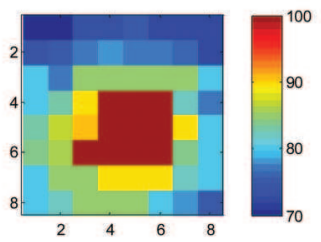

(e)

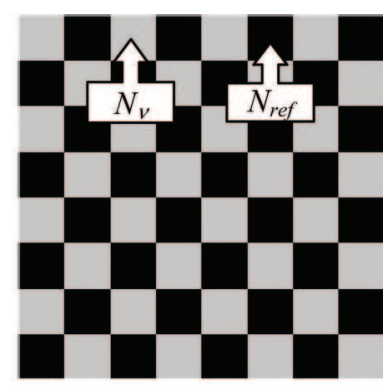

(b)

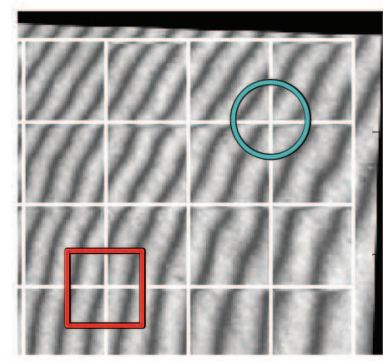

(d)

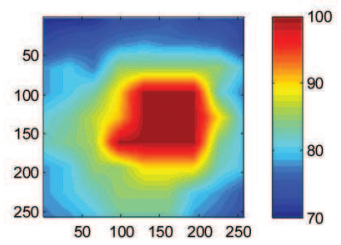

(f)
Fig. 7. (Color online) (a) Interferogram of BNS modulator with superimposed partition of $8 \times 8$ sectors. (b) Chessboard scheme used to determine $N_{2 \pi}(x, y)$ in each sector by fringe matching at the surroundings of the vertex. (c) Partial view of the interferogram for $N_{v}=70$. Fringes coincide at the border of the SLM aperture (circle). (d) Partial view of the interferogram for $N_{v}=100$. Fringes match at the central area of the aperture (square). (e) Map of $N_{2 \pi}(x, y)$ experimentally measured for $9 \times 9$ vertexes in the modulator aperture. (f) Interpolated map to the $256 \times 256$ pixels of the modulator aperture.

with the fringes of the diagonal neighbor cell [Figs. $7(\mathrm{c})-7(\mathrm{~d})]$. As an example, we can see that for a gray level $N_{v}=70$ there is a perfect match for fringes at the border of the SLM aperture [circle in Fig. 7(c)], whereas for $N_{v}=100$ the fringes coincide at the central region of the aperture [square in Fig. 7(d)]. Although the fringe matching was visually done it was very precise because of the high acuity of the human visual system to detect line mismatches (Vernier acuity [40]). A total of $9 \times 9$ vertex points were matched in the experiment. In Fig. 7(e) the $N_{2 \pi}$ map is depicted as it was sampled and measured. A smoothed version of the map obtained by interpolating it to the $256 \times 256$ pixels of the SLM is shown in Fig. 7(f).

Multipoint calibration can be done using either phase-shift interferometry, as we have just shown, or Fourier diffraction pattern analysis of binary phase gratings. We have repeated the multipoint calibra- 
tion using the latter method, that is, we have applied the method proposed by Zhang et al. [38] to each cell defined on the SLM aperture. We have searched the gray level value $N_{\pi}$ for which a phase shift of $\pi$ characterizes the binary phase grating displayed on the cell, and then the maximum intensity in the first order peak is reached. Using this method, Eq. (7) can be rewritten as

$$
\Delta \phi(x, y, N)=\Delta \phi_{\text {Norm }}(N) \frac{\pi}{\Delta \phi_{\text {Norm }}\left[N_{\pi}(x, y)\right]} .
$$

We measured the $N_{\pi}$ map and interpolated it to the $256 \times 256$ pixels of the SLM to build a smoothed version. The experimental results obtained in this case were very close to those obtained using phaseshift interferometry.

\section{Wavefront Distortion and Compensation}

Because of fabrication technology the LCD is not ideally optically flat. Reflective LCoS displays show a slight curvature, which is, in general, no more than a few waves. The silicon backplane curvature is one of the main contributors but, as we have seen in previous sections, the possible lack of spatial uniformity in the liquid crystal layer could be another one. Consequently, a phase distortion inherent to the device [30] is introduced in the wavefront. Depending on the application this has a minor effect, but in applications where an exhaustive wavefront control is required, the aberration needs to be compensated by the device itself using its phase modulation properties.

By means of the Michelson interferometer of Fig. 1, we register the interference fringe pattern between the wavefronts that come from the reference mirror and from the modulator. When the reference mirror is slightly tilted and a phase function equal to a constant gray level $N_{\text {ref }}=0$ is addressed to an ideally optical flat modulator, one expects to obtain a series of straight interference fringes as a consequence of the introduction of a linear phase ramp. However, Figs. 8(a) and 8(b) show the experimental interferograms registered for the Holoeye and BNS modulators, respectively. It can be seen that the fringes are not completely straight but bent lines because of the phase distortion introduced by the modulators. We analyzed the fringe series and rebuilt the respective wavefronts in Figs. 8(c) and 8(d). To remove the effect caused by the tilt of the reference mirror and isolate the wavefront reflected by the SLM, we decomposed the wavefronts of Figs. 8(c) and 8(d) using Zernike polynomials and suppressed the coefficients corresponding to tilts. We used the definition of the Zernike polynomials of the standard proposed by the Vision Science and its Applications Standards Task Force [41].

The fringe analysis allows us to determine the wavefront distortion (WFD), which is the difference between the maximum and the minimum values of the interferogram pattern expressed in $\lambda$ units. This parameter is useful to estimate the goodness of the compensation of the aberration. The compensation is

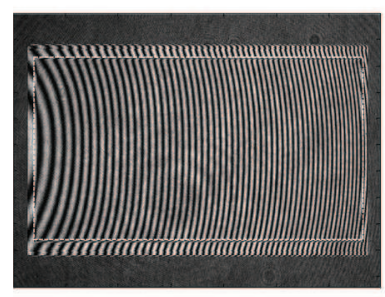

(a)

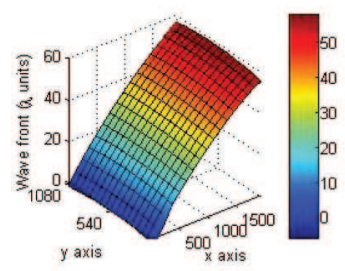

(c)

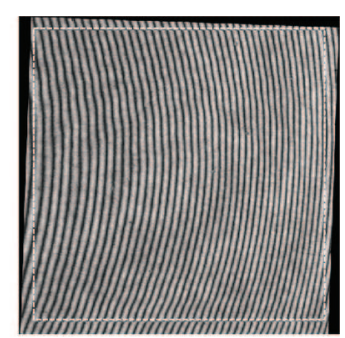

(b)

(d)

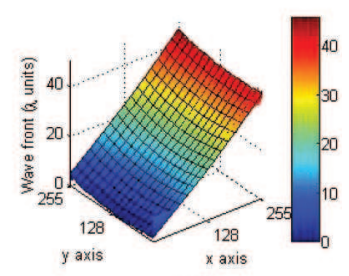

Fig. 8. (Color online) (a) Holoeye and (b) BNS SLM aperture interferograms recorded for a constant value of the gray level addressed to the modulator; (c) and (d) Wavefront distribution obtained respectively from (a) and (b).

based on the wavefront distortion described in terms of the Zernike polynomial coefficients. Since the Zernike decomposition is valid for a circular region and the SLM aperture is either square or rectangular, we calculate the WFD for both the inscribed circular aperture and the square aperture. In the case of the Holoeye SLM with rectangular aperture, we consider an anamorphically scaled aperture to convert it into a square. Consequently, the Zernike decomposition is actually defined on an elliptical basis for the Holoeye SLM.

We have compensated for the aberration of the wavefront by displaying a phase distribution consisting of different configurations of Zernike polynomials. We tried the first $6,10,15,21,28,36,45$, and 55 single index Zernike polynomials that correspond to the standard single indexing scheme that includes both radial and azimuthal parameters [41]. We also added a special configuration previously considered by Harriman et al. [20] named "20," that is, the combination of the single index polynomials from 0 to 17 , 24 , and 35 . After trying with a given compensation we registered the resulting interferogram and measured the WFD for both an inscribed circular region and the square aperture. In Fig. 9 the WFD is plotted versus the number of Zernike coefficients for both modulators. On the left of each figure, the WFD in the case of no compensation (denoted by $\mathrm{NC}$ in the abscissa axis) is also provided for the sake of comparison. For the Holoeye modulator [Fig. 9(a)], we see that the best option for the elliptical area is 28 and for the whole rectangular surface is 21 . For the BNS modulator [Fig. 9(b)], we see that the best configurations for the inscribed circular aperture are both 15 and " 20 ," whereas the best option to compensate the aberration in the whole square aperture is 15 . From the results shown in Fig. 9 we have chosen the best 


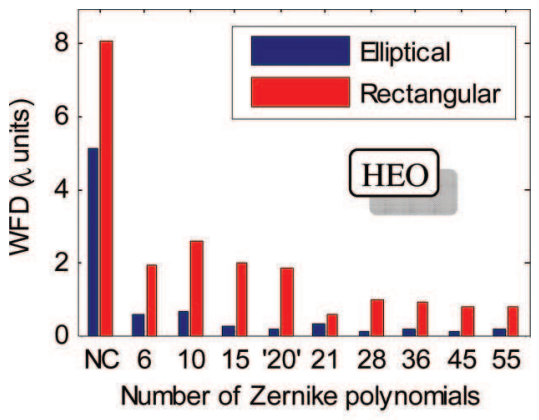

(a)

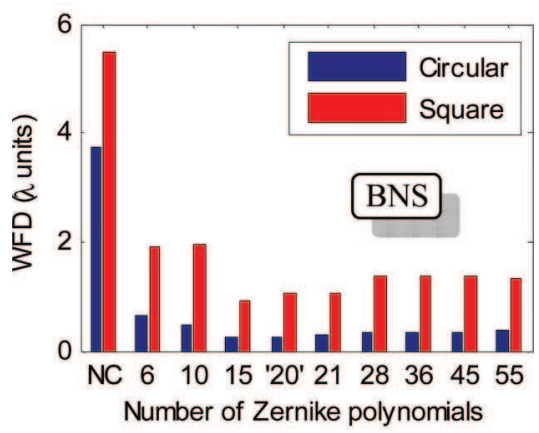

(b)

Fig. 9. (Color online) WFD computed using different decompositions of Zernike polynomials (coefficients according to single index notation), with no compensation (denoted by NC in the abscissa axis) and after compensation. (a) Holoeye 1080P SLM. In this case, the WFD was computed taking into account both an elliptical aperture and the whole rectangular aperture. (b) BNS SLM. The WFD was computed taking into account both the inscribed circular and the whole square aperture.

Zernike configuration for a circular (elliptical) aperture to compensate the aberration of each modulator. The interferograms obtained before and after the compensation of the wavefront are shown in Fig. 10 for the Holoeye modulator and in Fig. 11 for the BNS modulator. In the case of the compensation applied to the Holoeye modulator in Figs. 10(c) and 10(d), the global LUT of Fig. 3(c) was used to address the gray level distribution to the display. In the case of the compensation applied to the BNS modulator in Figs. 11(c) and 11(d), we used the multi-LUT built through the multipoint calibration described in Subsection 3.C. When the reference mirror of the Michelson interferomenter is not tilted, it can be seen that the WFD is less than $\lambda / 2$ in the central circular (elliptical) area of the modulators after compensation [Figs. 10 (c) and 11(c)]. On the other hand, when the reference mirror of the Michelson interferomenter is tilted, it can be seen that, after compensation, the interference fringes become straight lines as they correspond to a linear phase ramp [Figs. 10(d) and 11(d)].

In the experiment shown in Fig. 12 we analyze in more detail the relative importance of using either the global LUT or the multi-LUT to address a given phase distribution to the nonspatially uniform BNS modulator. Figure 12 presents the interferogram ob-

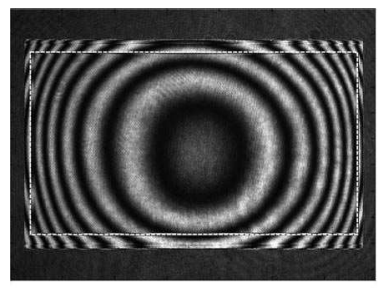

(a)

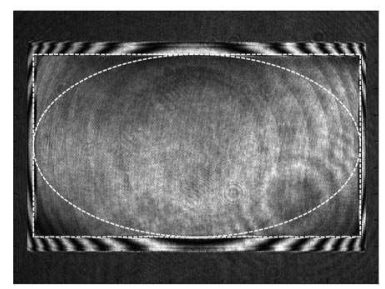

(c)

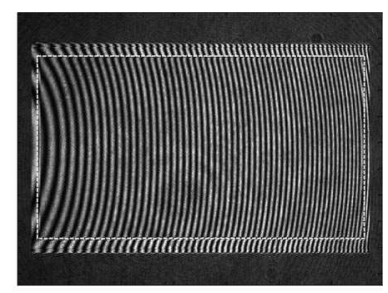

(b)

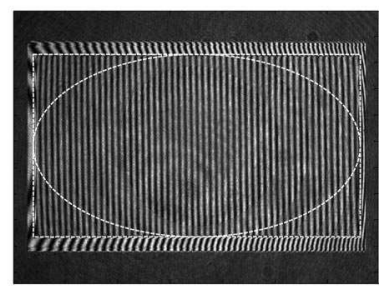

(d)
Fig. 10. Interferogram of the Holoeye modulator: (a) parallel reference mirror, without compensation of wavefront distortion; (b) tilted reference mirror, without compensation; (c) parallel mirror, with compensation of wavefront distortion; (d) tilted mirror, with compensation.

tained when a phase distribution corresponding to either X astigmatism (third Zernike polynomial) or $\mathrm{XY}$ astigmatism (fifth Zernike polynomial) is intended to be displayed on the BNS modulator. The ideal phase pattern distribution is also presented for comparison. It can be seen that if we apply the global LUT to address the phase distribution as it is initially

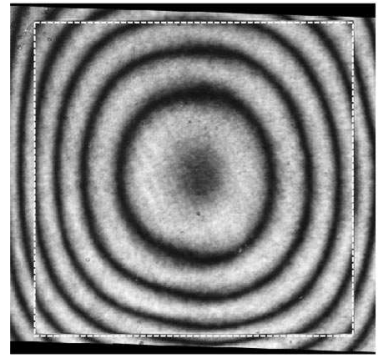

(a)

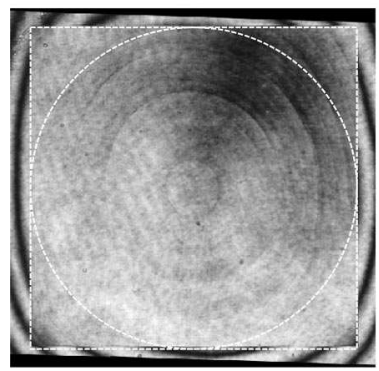

(c)

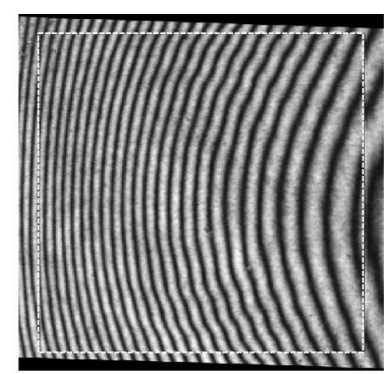

(b)

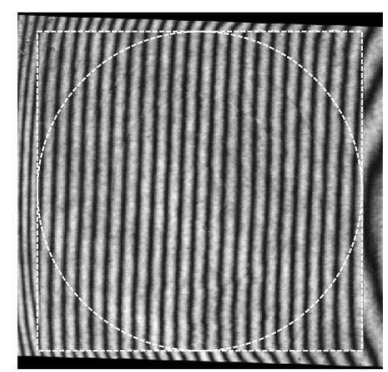

(d)
Fig. 11. Interferogram of BNS modulator: (a) parallel reference mirror, without compensation of wavefront distortion; (b) tilted reference mirror, without compensation; (c) parallel mirror, with compensation of wavefront distortion; (d) tilted mirror, with compensation. 

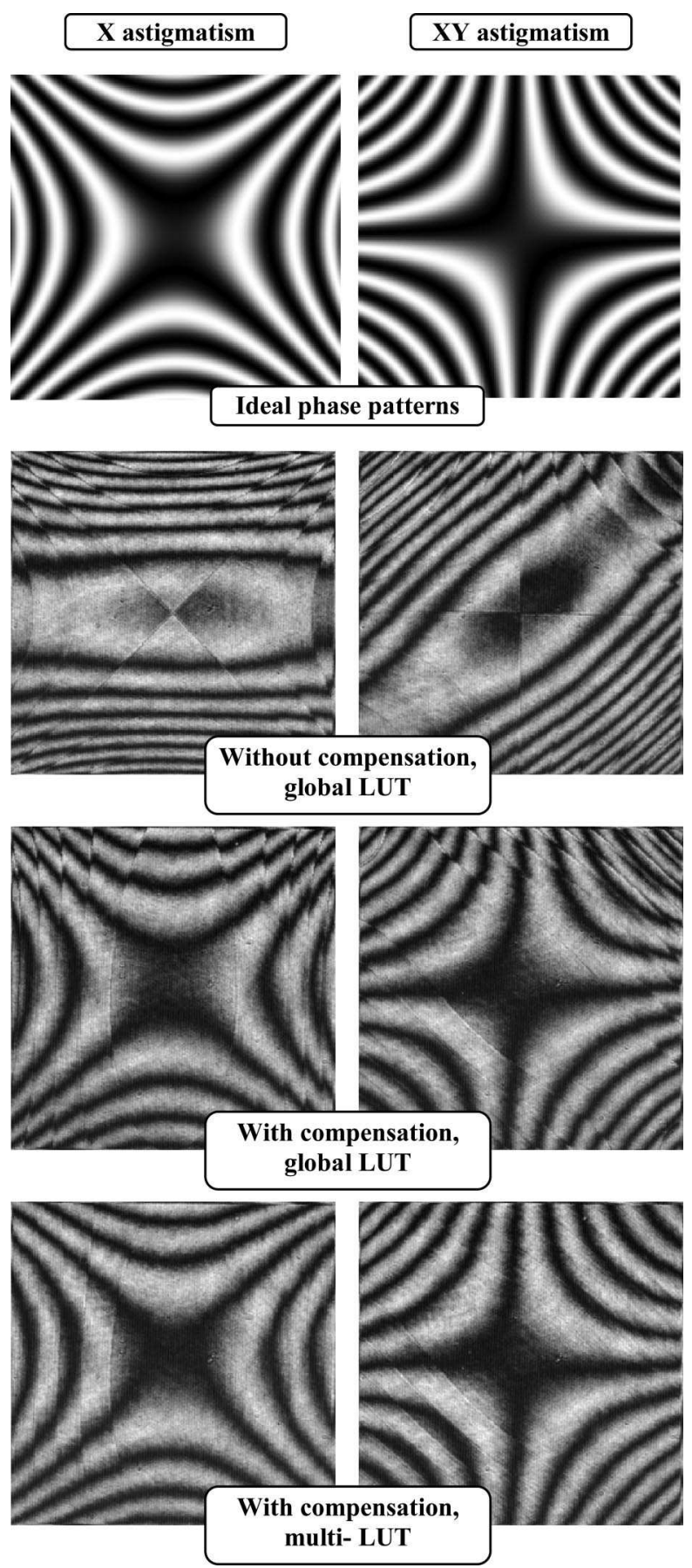

Fig. 12. Interferograms obtained when a phase distribution corresponding to either $\mathrm{X}$ astigmatism (3rd Zernike polynomial, column on the left) or XY astigmatism (5th Zernike polynomial, column on the right) is generated by the BNS modulator. Top: ideal phase pattern distributions; Second row: Without compensation, global LUT; Third row: With compensation, global LUT; Fourth row: With compensation, multi-LUT.

designed, without taking into account the compensation of wavefront distortion, that is, without applying the compensation given by the combination of the first 15 single index Zernike polynomials, then the result is far from the desired one. When the compensation of aberration is applied and the global LUT is used, the result resembles the desired one. However, some jagged fringes and discontinuities can be seen, particularly at the corners of the aperture. These artifacts disappear when we compensate for them after using the multi-LUT.

\section{Application to Diffractive Optical Elements}

Here we present an application for which the phase modulation improves after wavefront compensation along with the use of the proper LUT.

The reproduction of pure phase DOEs and computer generated holograms are highly efficient, with no complex conjugated component, minimized zero order, and high signal-to-noise ratio. To display DOEs on a parallel aligned LCD it is desirable that the SLM permits smooth phase modulation in the range $[0,2 \pi]$ and that the phase displayed on the device is nearly coincident with the computed phase. Otherwise, the DOE performance will be poor. The design and fabrication of DOEs were reviewed by Kress and Meyrueis [42].

We present the results obtained for a Fresnel hologram computed using the iterative Fourier transform algorithm [43] and displayed on both the Holoeye SLM (Fig. 13) and the BNS SLM (Fig. 14). A phase function of a lens was included in the hologram design. In the case of the Holoeye modulator the hologram was computed to a resolution of $512 \times 512$ pixels, and then duplicated to fill the resolution of $1024 \times 1024$ pixels to reduce the speckle noise. The focal length of the lens was $f^{\mathrm{HEO}}=100 \mathrm{~cm}$ for practical purposes. In the case of the BNS modulator, the hologram was computed for $128 \times 128$ pixels and also duplicated to fill the resolution of $256 \times 256$ pixels. The focal length was $f_{\text {ref }}^{\text {BNS }}=13.1 \mathrm{~cm}$. This focal length corresponds to the lens that best suits the

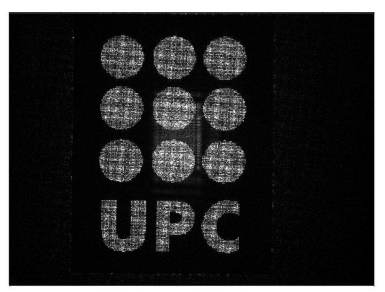

(a)

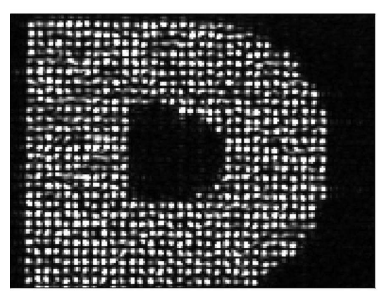

(b)

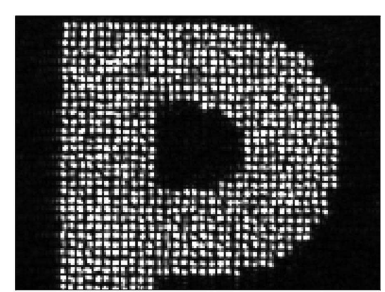

(c)
Fig. 13. Reconstruction of a Fresnel hologram of the UPC logo using the Holoeye SLM, with a focal length lens of $f=100 \mathrm{~cm}$ included in the hologram design. (a) Overview of image reconstruction; (b) (detail) without compensation; (c) (detail) with compensation. 


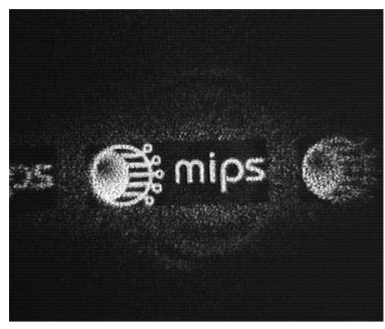

(a)

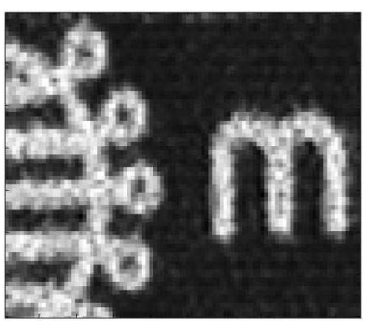

(c)

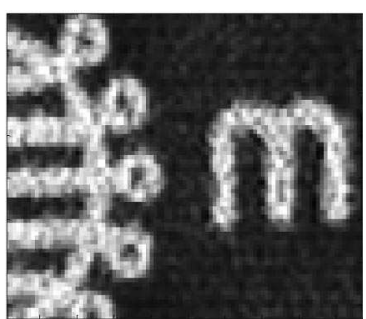

(b)

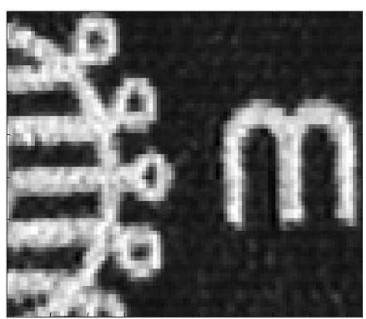

(d)
Fig. 14. Reconstruction of a Fresnel hologram of the MIPS logo using the BNS SLM, with a focal length lens of $f=13.1 \mathrm{~cm}$ included in the hologram design. (a) Overview of image reconstruction; (b) (detail) without compensation of the aberration and using the global LUT; (c) without compensation and using the multiLUT; (d) with compensation and using the multi-LUT.

modulator characteristics (resolution and pixel size) and the illuminating wavelength [6].

The absence of the complex conjugated and the very weak peak at zero order are evidence of the quality of the phase coding of the holograms shown in Figs. 13 and 14. The algorithm used to compute the holograms is very robust to small errors. The second and higher orders of image reconstruction, visible in Fig. 14(a), are caused by the diffraction grid of the pixelated structure of the liquid crystal device.

Figure 13(a) shows a general view of the reconstructed hologram of the UPC logo by using the Holoeye modulator. Detailed views of this hologram, without compensation of aberration and with compensation, are shown in Figs. 13(b) and 13(c), respectively. The global LUT of Fig. 3(c) was used to address the gray level distribution to the display. The camera was adjusted to capture the best focal plane. The quality of the hologram is improved when compensation of the aberration is introduced.

With regard to the quality of the images reconstructed in Fig. 14 it can be seen that the compensation for aberration introduced by the BNS modulator is important. If no compensation is introduced [Figs. 14(b) and 14(c)], then the focalization plane changes. This change cannot be seen in Fig. 14 because the position of the camera was adjusted to the best focus plane. The best quality is obtained when both the phase compensation and the multi-LUT are considered [Fig. 14(d)]. However, since holograms are redundant, they are not so sensitive to the encoding errors. Although the image quality is affected in the hologram reconstruction, the use of the multi-LUT does not seem to be critical. This is not the case for applications such as wavefront generation or adaptive optics, for which the quality of the wavefront must be high as shown in Fig. 12.

\section{Conclusions}

The experimental characterization of a parallel aligned LCoS spatial light modulator in terms of phase modulation versus the addressed gray level and inherent wavefront distortions is essential for the correct use of the modulator in applications with high requirements of the wavefront control. The modulator performance considerably improves by introducing a phase pattern that self-compensates for inherent wavefront distortions and that is added to the programmed phase distribution. The calibration of the phase modulation capability determines the effective range of phase modulation and tests linearity versus the addressed gray level (voltage). From this experimentation it is necessary to build an LUT to map the range of addressable gray levels (e.g., 8 -bits) to the phase modulation range $[0,2 \pi]$ and linearize the modulator response. Different techniques have experimentally been used in this work to calibrate the phase modulation capability of two reflective parallel aligned LCoS SLMs. The modulator with a spatially uniform response was calibrated by fringe analysis using an interferometer. An LUT was built for the effective display of a phase pattern on this modulator. However, this method could not be applied to a modulator with a nonuniform response through its aperture. In such a case, we used a method based on displaying binary phase grating and analyzing the intensity of the first diffraction order of the Fourier spectrum. This method allowed us to determine the average phase modulation capability of the device and build a global LUT. This global LUT permits us to approximately display the phase function addressed to the modulator. But because of the lack of spatial uniformity of the device (for example, with higher phase modulation capability in the borders than in the center of the BNS SLM aperture), a programmed phase pattern can only be reproduced in first approach, even though it may have been compensated for aberration. The method proposed here develops an exhaustive multipoint calibration of the spatially nonuniform SLM. It leads to building a multi-LUT for more precise control of the modulator response, particularly at the corners of the aperture. In the experimental results and the application on DOE generation, the compensation of the wavefront distortion is highly convenient and, in the case of having a spatially nonuniform SLM, the use of the multi-LUT improves the device performance in comparison with the global LUT.

The authors are grateful to Laurent Bigué for many fruitful discussions. This research was partly supported by CNRS, Département STIC (EPML 8 fundings), and by the Spanish Ministerio de Educación y Ciencia and FEDER funds (DPI2006-05479). 
Pierre Ambs was partly supported by the European project INTERREG III Rhenaphotonics. Joaquín Otón acknowledges a scholarship from the Spanish Ministerio de Educación y Ciencia.

\section{References}

1. D. C. O'Brien, R. J. Mears, T. D. Wilkinson, and W. A. Crossland, "Dynamic holographic interconnects that use ferroelectric liquid crystal spatial light modulators," Appl. Opt. 33, 2795-2803 (1994).

2. D. C. O'Brien, G. E. Faulkner, T. D. Wilkinson, B. Robertson, and D. G. Leyva, "Design and analysis of an adaptive boardto-board dynamic holographic interconnect," Appl. Opt. 43, 3297-3305 (2004).

3. J. A. Davis, D. M. Cottrell, R. A. Lilly, and S. W. Connely, "Multiplexed phase-encoded lenses written on spatial light modulators," Opt. Lett. 14, 420-422 (1989).

4. V. Laude, "Twisted-nematic liquid crystal pixelated active lens," Opt. Commun. 153, 134-152 (1998).

5. M. J. Yzuel, J. Campos, A. Marquez, J. C. Escalera, J. A. Davis, C. Lemmi, and S. Ledesma, "Inherent apodization of lenses encoded on liquid crystal spatial light modulators," Appl. Opt. 39, 6034-6039 (2000).

6. J. Otón, M. S. Millán, and E. Pérez-Cabré, "Programmable lens design in a pixelated screen of twisted-nematic liquid crystal display," Opt. Pura Apl. 38, 47-56 (2005).

7. J. Campos, A. Marquez, M. J. Yzuel, J. A. Davis, D. M. Cottrell, and I. Moreno, "Fully complex synthetic discriminant functions written onto phase-only modulators," Appl. Opt. 39, 5965-5970 (2000).

8. P. J. Rodrigo, R. L. Eriksen, V. R. Daria, and J. Glückstad, "Shack-Hartmann multiple-beam optical tweezers," Opt. Express 11, 208-214 (2003).

9. M. Reicherter, T. Haist, E. U. Wagemann, and H. J. Tiziani, "Optical particle trapping with computer-generated holograms written on a liquid-crystal display," Opt. Lett. 24, 608-610 (1999).

10. H. J. Tiziani, T. Haist, J. Liesener, M. Reicherter, and L. Seifert, "Applications of SLMs for optical metrology," Proc. SPIE 4457, 72-81 (2001).

11. W. Osten, C. Kohler, and J. Liesener, "Evaluation and application of spatial light modulators for optical metrology," Opt. Pura Apl. 38, 71-81 (2005).

12. A. Michalkiewicz, M. Kujawinska, T. Kozacki, X. Wang, and P. J. Bos, "Holographic three-dimensional displays with liquid crystal on silicon spatial light modulator," Proc. SPIE 5531, 85-94 (2004).

13. M. Stanley, M. A. Smith, A. P. Smith, P. J. Watson, S. D. Coomber, C. D. Cameron, C. W. Slinger, and A. D. Wood, "3D electronic holography display system using a 100 megapixel spatial light modulator," Proc. SPIE 5249, 297-308 (2004).

14. T. Shirai and T. H. Barnes, "Adaptive restoration of a partially coherent blurred image using an all-optical feedback interferometer with a liquid crystal device," J. Opt. Soc. Am. A 19, 369-377 (2002).

15. S. P. Laut, D. U. Bartsch, and W. R. Freeman, "Experimental approach to the characterization of a micromachined continuous-membrane deformable mirror," Proc. SPIE 5169, 95-103 (2003).

16. P. M. Prieto, E. J. Fernández, S. Manzanera, and P. Artal, "Adaptive optics with a programmable phase modulator: applications in the human eye," Opt. Express 12, 4059-4071 (2004).

17. A. Márquez, C. Iemmi, J. Campos, J. Escalera, and M. Yzuel, "Programmable apodizer to compensate chromatic aberration effects using a liquid crystal spatial light modulator," Opt. Express 13, 716-730 (2005).

18. M. S. Millán, J. Otón, and E. Pérez-Cabré, "Chromatic compensation of programmable Fresnel lenses," Opt. Express 14, 6226-6242 (2006).

19. M. S. Millán, J. Otón, and E. Pérez-Cabré, "Dynamic compensation of chromatic aberration in a programmable diffractive lens," Opt. Express 14, 9103-9012 (2006).

20. J. Harriman, A. Linnenberger, and S. Serati, "Improving spatial light modulator performance through phase compensation," Proc. SPIE 5553, 58-67 (2004).

21. B. E. A. Saleh and K. Lu, "Theory and design of the liquid crystal TV as an optical spatial phase modulator," Opt. Eng. 29, 240-246 (1990).

22. C. Soutar and K. Lu, "Determination of the physical properties of an arbitrary twisted-nematic liquid crystal cell," Opt. Eng. 33, 2704-2712 (1994).

23. J. A. Davis, I. Moreno, and P. Tsai, "Polarization eigenstates for twisted-nematic liquid crystal displays," Appl. Opt. 37, 937-945 (1998).

24. I. Moreno, J. A. Davis, K. G. D’Nelly, and D. B. Allison, “Transmission and phase measurement for polarization eigenvectors in twisted-nematic liquid crystal spatial light modulators," Opt. Eng. 37, 3048-3052 (1998).

25. J. A. Davis, D. B. Allison, K. G. D’Nelly, M. L. Wilson, and I. Moreno, "Ambiguities in measuring the physical parameters for twisted-nematic liquid crystal spatial light modulators," Opt. Eng. 38, 705-709 (1999).

26. A. Márquez, J. Campos, M. J. Yzuel, I. Moreno, J. A. Davis, C. Iemmi, A. Moreno, and A. Robert, "Characterization of edge effects in twisted nematic liquid crystal displays," Opt. Eng. 39, 3301-3307 (2000).

27. V. Duran, J. Lancis, E. Tajahuerce, and Z. Jaroszewicz, "Univocal determination of the cell parameters of a twisted nematic liquid crystal display by single-wavelength polarimetry," J. Appl. Phys. 97, 043101 (2005).

28. D. J. Cho, S. T. Thurman, J. T. Donner, and G. M. Morris, "Characteristics of a $128 \times 128$ liquid crystal spatial light modulator for wavefront generation," Opt. Lett. 23, 969-971 (1998).

29. I. Moreno, C. Iemmi, A. Márquez, J. Campos, and M. J. Yzuel, "Modulation light efficiency of diffractive lenses displayed in a restricted phase-mostly modulation display," Appl. Opt. 43, 6278-6284 (2004).

30. X. Xun and R. W. Cohn, "Phase calibration of spatially nonuniform spatial light modulators," Appl. Opt. 43, 6400-6406 (2004).

31. J. Otón and P. Ambs, "Characterization and applications of a pure phase reflective liquid crystal spatial light modulator," Proc. SPIE 6254, 62540N (2006)

32. P. Grother and D. Casasent, "Optical path difference measurement technique for SLMs," Opt. Commun. 189, 31-38 (2001).

33. J. D. Downie, B. P. Hine, and M. B. Reid, "Effects and correction of magneto-optic spatial light modulator phase errors in an optical correlator," Appl. Opt. 31, 636-643 (1992).

34. A. J. Bergeron, F. Gauvin, D. Gagnon, H. Gingras, H. H. Arsenault, and M. Doucet, "Phase calibration and applications of a liquid crystal spatial light modulator," Appl. Opt. 34, 5133-5139 (1995).

35. R. Dou and M. K. Giles, "Simple technique for measuring the phase property of a twisted nematic liquid crystal television," Opt. Eng. 35, 808-812 (1996).

36. Holoeye Photonics AG and Holoeye Corporation $\langle\mathrm{http}: / / \mathrm{www}$. holoeye.com $\rangle$.

37. D. Malacara, ed., Optical Shop Testing, 2nd ed. (Wiley, 1992), Chap. 14, p. 501.

38. Z. Zhang, G. Lu, and F. Yu, "Simple method for measuring 
phase modulation in liquid crystal television," Opt. Eng. 33, 3018-3022 (1994).

39. Boulder Nonlinear Systems 〈http://www.bnonlinear.com〉.

40. A. G. Bennett and R. B. Rabbetts, Clinical Visual Optics, 3rd ed. (Butterworth-Heinemann, 1998).

41. L. N. Thibos, R. A. Applegate, J. T. Schwiegerling, and R. Webb, "Standards for reporting the optical aberrations of eyes," in Vision Science and Its Applications, Vol. 35 of Trends in Optics and Photonics Series, V. Lakshminarayanan, ed. (Optical Society of America, 2000), 232-244.

42. B. Kress and P. Meyrueis, Digital Diffractive Optics, an Introduction to Planar Diffractive Optics and Related Technology (Wiley, 2000).

43. F. Wyrowski and O. Bryngdahl, "Iterative Fourier-transform algorithm applied to computer holography," J. Opt. Soc. Am. A 5, 1058-1065 (1988). 